\title{
Symptoms of Autonomic Dysfunction in Systemic Sclerosis Assessed by the COMPASS-31 Questionnaire
}

\author{
Brittany L. Adler, James W. Russell, Laura K. Hummers, and Zsuzsanna H. McMahan
}

ABSTRACT. Objective. Autonomic dysfunction is a known complication of systemic sclerosis (SSc) that can affect vascular tone, gastrointestinal (GI) motility, heart rate, and blood pressure control. We sought to quantify autonomic symptom burden in SSc, and to define the characteristics of patients with SSc and autonomic dysfunction.

Methods. Patients with SSc were consecutively recruited during routine clinical visits at the Johns Hopkins Scleroderma Center and asked to complete the Composite Autonomic Symptom Score (COMPASS)-31 questionnaire, a validated tool to assess symptoms of autonomic dysfunction. We determined the relationship between various clinical and serological features of SSc and the total COMPASS-31 scores and domain-specific scores using the Student t test or Wilcoxon rank-sum test for dichotomous variables and linear regression analysis for continuous variables.

Results. The study included 104 patients with SSc who completed the COMPASS-31 questionnaire. The mean COMPASS-31 score in this cohort was $24.9 \pm 15.5$, higher than COMPASS-31 scores from previously published healthy controls $(8.9 \pm 8.7)$. Compared to patients with mild or absent GI disease, patients with significant GI disease had higher scores across several subdomains of the COMPASS-31, including orthostatic intolerance (median $10.0 \mathrm{vs} 0, \mathrm{p}=0.006$ ) and secretomotor dysfunction (median 6.4 vs $4.3, \mathrm{p}=0.03$ ). There was also a dose-response relationship between GI disease severity and autonomic symptom burden.

Conclusion. Symptoms of autonomic dysfunction are common in SSc. Patients with more severe GI disease in SSc report more symptoms of dysautonomia across many facets of the autonomic nervous system. (First Release June 15 2018; J Rheumatol 2018;45:1145-52; doi:10.3899/jrheum.170868)

Key Indexing Terms:

GASTROINTESTINAL TRACT

NEUROLOGIC MANIFESTATIONS SELF-ASSESSMENT AUTONOMIC DYSFUNCTION

\footnotetext{
From the Department of Rheumatology, Johns Hopkins University School of Medicine; the departments of Neurology, Anatomy, and Neurobiology, University of Maryland School of Medicine; the Veterans Affairs Medical Center, Baltimore, Maryland, USA.

Supported by the Rheumatology Research Foundation Scientist

Development Award, the Jerome L. Greene Foundation (90057213), the Scleroderma Research Foundation (ZHM), the Johns Hopkins Clinician Scientist Research Career Development Award (ZHM), and the US National Institute of Arthritis and Musculoskeletal and Skin Diseases (BLA, T32AR048522). JWR was supported in part by the US National Institute of Diabetes and Digestive and Kidney Diseases, the National Institutes of Health and Office of Research Development (1R01DK107007-01A1), the Department of Veterans Affairs (Biomedical and Laboratory Research Service and Rehabilitation Research and Development, 101RX001030).

B.L. Adler, MD, Rheumatology Fellow, Department of Rheumatology, Johns Hopkins University School of Medicine; J.W. Russell, MD, Professor of Neurology, departments of Neurology, Anatomy and Neurobiology, University of Maryland School of Medicine and Veterans Affairs Medical Center; L.K. Hummers, MD, MSc, Associate Professor of Medicine, Department of Rheumatology, Johns Hopkins University School of Medicine; Z.H. McMahan, MD, MHS, Assistant Professor of Medicine, Department of Rheumatology, Johns Hopkins University School of Medicine.

Drs. Hummers and McMahan contributed equally to this work. Author correspondence to Dr. B.L. Adler, 5200 Eastern Ave., MFL Center Tower, Suite 4100, Baltimore, Maryland 21224, USA.

E-mail: badler6@jhu.edu

Accepted for publication March 2, 2018.
}

Systemic sclerosis (SSc) is an autoimmune multiorgan disease associated with significant morbidity due to organ dysfunction ${ }^{1}$. Pathological studies demonstrate microvascular disease and/or tissue fibrosis involving the skin, lungs, heart, kidney, gastrointestinal (GI) tract, and muscles ${ }^{2,3}$. Dysregulation of the autonomic nervous system (dysautonomia) can occur early or late in the disease $e^{4,5,6}$. In some cases, dysautonomia results in abnormal vascular tone $\mathrm{e}^{7,8}$, GI dysmotility ${ }^{9,10}$, and dysregulation of heart rate and blood pressure $^{11}$.

The mechanisms underlying autonomic dysfunction in SSc remain unclear, but could be autoimmune damage to nerves, vascular disease, or direct nerve compression from tissue fibrosis ${ }^{12}$. Tissue fibrosis is unlikely to be the only cause of autonomic dysfunction in SSc, however, because degeneration of the autonomic neural network and ganglion root cells have been observed in areas of unaffected $\operatorname{skin}^{12,13,14}$. Autonomic dysfunction is often seen early in the disease course, suggesting that it may play an important pathological role in SSc.

Although dysautonomia is recognized to occur in a subset of patients with SSc, the prevalence of symptoms related to autonomic dysfunction and the clinical features of SSc

Personal non-commercial use only. The Journal of Rheumatology Copyright @ 2018 . All rights reserved. 
associated with dysautonomia remain undefined. Understanding the disease features of SSc that are associated with dysautonomia may provide important insights into the underlying disease mechanism, and may also improve recognition and diagnosis of this complication in clinical practice.

The Composite Autonomic Symptom Score (COMPASS)-31 is a self-administered 31-item questionnaire that quantifies self-reported autonomic symptoms ${ }^{15}$. It is an abbreviated version of the 164-item COMPASS assessment tool ${ }^{16}$. The COMPASS-31 was validated in a cohort of patients with small fiber neuropathy ${ }^{17}$, and was previously used to quantify self-reported autonomic symptoms in multiple sclerosis ${ }^{18}$, diabetic neuropathy ${ }^{19}$, and fibromyalgia ${ }^{20}$. In our present study, we used the COMPASS-31 to quantify the frequency and features of autonomic symptoms in a cohort of patients with SSc.

\section{MATERIALS AND METHODS}

Study population. All patients were seen for routine clinical evaluations at the Johns Hopkins Scleroderma Center between March 2016 and April 2016. Patients were consecutively recruited after their clinical visits if they met either the 2013 American College of Rheumatology/ European League Against Rheumatism (ACR/EULAR) criteria for SSc, the 1980 ACR criteria, or had at least 3 of 5 features of the CREST syndrome [calcinosis, Raynaud phenomenon (RP), esophageal dysmotility, sclerodactyly, telangiectasias]. Patients were categorized by extent of skin disease as limited or diffuse SSc based on previously established criteria ${ }^{21}$.

Healthy control population. We compared COMPASS-31 scores in our SSc cohort to scores in healthy controls and other populations with autonomic dysfunction in the published literature ${ }^{17,19,20}$. The healthy control cohort consisted of 30 female participants who were healthy and free of pain and fatigue (Vincent, et $a l^{20}$ has cohort details).

Assessment tool: COMPASS-31 questionnaire. The COMPASS-31 consists of 31 questions that fall into 6 domains of dysautonomia: orthostatic intolerance (4 items), vasomotor dysfunction (3 items), secretomotor dysfunction (4 items), GI dysfunction (12 items; includes gastroparesis, constipation, and diarrhea), urinary dysfunction ( 3 items), and pupillomotor dysfunction (5 items). An answer was scored as zero when it was not assigned a point. A raw domain score was obtained by adding together points within each domain. The total score within each domain was weighted as previously described ${ }^{15}$ and then added together to give a total score ranging from 0 to 100. The maximum weighted scores for each subdomain are as follows: 40 for orthostatic intolerance, 5 for vasomotor dysfunction, 15 for secretomotor dysfunction, 25 for GI dysfunction, 10 for urinary dysfunction, and 5 for pupillomotor dysfunction.

Clinical data collection. Demographic and clinical data for all study participants were obtained from the Johns Hopkins Scleroderma Center longitudinal database. The center's database includes date of birth, sex, race, date of onset of first RP and first non-RP symptoms, medications, and various measures of disease severity and clinical features obtained at the first clinic visit and at 6-month intervals during followup visits. Medications that the patient was taking at the time of completion of the COMPASS-31 questionnaire were used for analysis. Of particular interest was the use of a blood pressure medication or vasodilator, because these medications can cause orthostatic symptoms as a side effect. We also recorded the patient heart rate by taking the average of the heart rate at the time the COMPASS-31 questionnaire was completed with the heart rates measured at the immediate prior and subsequent clinical visit.

The presence and severity of organ involvement were assessed using the maximum Medsger severity score ${ }^{22}$ recorded across all longitudinal visits. Because clinical features of SSc are known to change over time, the most extreme data points recorded ( $\mathrm{max} / \mathrm{min}$, ever/never) were used to fully identify disease manifestations. The degree of skin involvement was scored using the maximum modified Rodnan skin score (mRSS; range 0-51). Significant GI disease was defined by a Medsger severity score $\geq 2(0=$ no symptoms; 1 = acid reflux medications or abnormal small bowel series; 2 = high-dose acid reflux medications or antibiotics for bacterial overgrowth; 3 = diagnosis of malabsorptive syndrome or episodes of pseudo-obstruction; $4=$ dependence on total parenteral nutrition). Severe GI disease was defined as a Medsger severity score of 3 or 4 . Significant cardiac disease was defined by a Medsger score $\geq 1$, and significant lung disease and RP were defined by a Medsger score $\geq 2$. The presence of myopathy was defined by the presence of muscle weakness (Medsger score $\geq 1$ defined by power $\leq 4 / 5$ in the upper or lower extremities) as well as the presence of at least 1 of the following: elevation in creatinine phosphokinase, abnormal electromyography, muscle edema visualized on magnetic resonance imaging, or myopathy confirmed on muscle biopsy. Also recorded were the presence of arthralgia, arthritis, dry eyes or dry mouth, or history of SSc renal crisis, digital ulceration/gangrene, and right heart catheterization-confirmed pulmonary arterial hypertension (defined as a mean pulmonary artery pressure $\geq 25 \mathrm{mmHg}$ and pulmonary capillary wedge pressure $\leq 15 \mathrm{mmHg}$ ).

Written informed consent was obtained from all patients. This study was approved by the Johns Hopkins Institutional Review Board (approval number 00034985)

Autoantibody analysis. A commercially available line immunoblot assay [Scleroderma (Nucleoli) Profile Euroline (IgG); Euroimmun] was used to determine the SSc autoantibody profiles for patients with available serum. Assays and analysis were performed by a single operator per the manufacturer's instructions as previously described ${ }^{23}$. Readings were considered as defined by the assay (positive or negative), and results in the borderline range were considered negative.

Statistical analysis. COMPASS-31 scores were compared across dichotomous clinical and demographic variables using the Student $t$ test for scores that were normally distributed (total COMPASS-31 score, vasomotor dysfunction, GI dysfunction, pupillomotor dysfunction) and the Wilcoxon rank-sum test for scores that were not normally distributed (orthostatic intolerance, secretomotor dysfunction, and urinary dysfunction). Results are depicted as mean $\pm \mathrm{SD}$ or median (interquartile range; IQR). Linear regression analyses were used to determine the relationship between continuous clinical independent variables and COMPASS-31 scores (dependent variable). Multivariable linear regression models were used to adjust for possible covariates, including age, sex, race, and any variables that are associated with the COMPASS-31 score.

To determine whether the associations between disease features and severity and the total COMPASS-31 score resulted from global autonomic symptoms across several subdomains, we grouped patients by the number of positive subdomains (score $>0$ in any category) of the COMPASS-31. We divided the cohort into 2 groups based on the graphical distribution of the data prior to the analysis: (1) the global autonomic symptoms group, which included all patients with 5 or more positive subdomains; and (2) the limited autonomic symptoms group, which included patients with fewer than 5 positive subdomains. Using a chi-square analysis, we determined the relationship between the presence of specific clinical features of SSc and global autonomic dysfunction. Multivariable logistic regression models were used to adjust for clinically relevant covariates and potential confounders.

All statistical analyses were conducted using JMP Version 9 (SAS Institute Inc.). All statistical tests were 2-sided, and statistical significance was defined as $\mathrm{p}<0.05$ for all analyses.

\section{RESULTS}

Patient characteristics. The study included 104 consecutive patients with SSc who were recruited and completed the COMPASS-31 questionnaire. Demographic information for all study participants is shown in Table 1 . The mean age was 
Table 1. Demographics and disease characteristics of patients with systemic sclerosis.

\begin{tabular}{|c|c|}
\hline Characteristics & Values \\
\hline Age, yrs, mean (SD) & $57(12.6)$ \\
\hline \multicolumn{2}{|l|}{ Sex } \\
\hline Female, n (\%) & $92(88)$ \\
\hline Male, n (\%) & $12(12)$ \\
\hline \multicolumn{2}{|l|}{ Race, n (\%) } \\
\hline White & $77(74)$ \\
\hline African American & $17(16)$ \\
\hline \multicolumn{2}{|l|}{ SSc type, n (\%) } \\
\hline Limited & $56(54)$ \\
\hline Diffuse & $48(46)$ \\
\hline \multicolumn{2}{|l|}{ Disease duration, yrs } \\
\hline From onset of RP, median (IQR) & $12.5(7-22)$ \\
\hline From onset of non-RP symptom, median (IQR) & $11.0(7-18)$ \\
\hline No. with disease duration $<3$ yrs from RP, n $(\%)$ & $12(12)$ \\
\hline No. with disease duration $<5$ yrs from RP, $\mathrm{n}(\%)$ & $18(17)$ \\
\hline \multicolumn{2}{|l|}{ Antibody status, $\mathrm{n} / \mathrm{N}(\%)$} \\
\hline Anti-centromere & $21 / 83(25)$ \\
\hline Anti-topoisomerase I & $31 / 83(37)$ \\
\hline Anti-RNA-polymerase III & $19 / 83(23)$ \\
\hline Anti-PM-Scl & $12 / 83(15)$ \\
\hline Anti-U3-RNP & $8 / 83(10)$ \\
\hline Anti-Ro-52 & $19 / 83(23)$ \\
\hline Anti-ThTo & $6 / 83(7)$ \\
\hline Anti-Ku & $4 / 83(5)$ \\
\hline \multicolumn{2}{|l|}{ Clinical features } \\
\hline Calcinosis, $\mathrm{n}(\%)$ & $51(49)$ \\
\hline Arthralgias, n (\%) & $92(88)$ \\
\hline Synovitis, $\mathrm{n}(\%)$ & $25(24)$ \\
\hline Digital ulceration or gangrene, $\mathrm{n}(\%)$ & $27(26)$ \\
\hline Scleroderma renal crisis, $\mathrm{n}(\%)$ & $4(4)$ \\
\hline Myopathy, n (\%) & $16(15)$ \\
\hline Pulmonary arterial hypertension ${ }^{\diamond}, \mathrm{n}(\%)$ & $7(7)$ \\
\hline Maximum RVSP (mmHg), mean (SD) & $39.6(12.2)$ \\
\hline Modified Rodnan skin score, mean (SD) & $13.8(12.0)$ \\
\hline Minimum FVC, mean (SD) & $72.7(21.1)$ \\
\hline Minimum DLCO, mean (SD) & $65.3(23.7)$ \\
\hline Significant RP*, n (\%) & $59(57)$ \\
\hline Significant lung disease*, n (\%) & $68(65)$ \\
\hline Significant heart disease ${ }^{\dagger}, \mathrm{n}(\%)$ & $27(26)$ \\
\hline Significant GI disease*, $\mathrm{n}(\%)$ & $64(62)$ \\
\hline
\end{tabular}

${ }^{\diamond}$ Pulmonary arterial hypertension was defined as a mean pulmonary artery pressure $\geq 25 \mathrm{mmHg}$ and a pulmonary capillary wedge pressure $\leq 15 \mathrm{mmHg}$. * Significant RP, lung disease, and GI disease were defined as a Medsger severity score $\geq 2$. ${ }^{\dagger}$ Significant heart disease was defined as a Medsger severity score $\geq 1$. Right heart catheterization data were available for 20 patients. SSc: systemic sclerosis; RVSP: right ventricular systolic pressure; FVC: forced vital capacity; IQR: interquartile range; RP: Raynaud phenomenon; GI: gastrointestinal

$57 \pm$ SD 12.6 years, $88 \%$ of the cohort was female, $74 \%$ were white, and $16 \%$ were African American. The median disease duration from the onset of RP was 12.5 years (IQR 7-22) and the median disease duration from the onset of a non-RP symptom of SSc was 11.0 years (IQR 7-18). The majority of patients $(54 \%)$ had limited cutaneous disease and $46 \%$ had diffuse cutaneous disease.
Patients with SSc have high COMPASS-31 scores that are comparable to scores among patients with other disorders known to present with dysautonomia. The mean COMPASS-31 score in our SSc cohort was $24.9 \pm 15.5$. We assessed whether this COMPASS-31 score was similar to COMPASS-31 scores from healthy controls and patients with nonrheumatic diseases that present with dysautonomia ${ }^{17,19,20}$ (Table 2). We found that patients with SSc had higher COMPASS-31 scores compared to a previously published healthy control population ${ }^{20}(24.9 \pm 15.5$ for SSc vs $8.9 \pm 8.7$ for healthy controls), and the differences in scores spanned across several domains of autonomic function. Mean COMPASS-31 scores in SSc were comparable to other diseases that present with autonomic dysfunction, including diabetic polyneuropathy and small-fiber polyneuropathy ${ }^{17,19}$.

Given that many patients with SSc are treated with vasodilators, which can cause orthostatic symptoms as a side effect, we examined whether the use of these medications may affect the COMPASS-31 score. We did not find a difference in the mean COMPASS-31 score or orthostatic intolerance score between patients treated with a blood pressure or vasodilator medication $(n=67)$ and those who were untreated $(n=37, p>0.05)$. We next evaluated whether skin fibrosis may directly affect sweat glands and therefore result in more secretomotor dysfunction in SSc. We did not find an association between the maximum mRSS and the secretomotor dysfunction score $(\mathrm{p}>0.05)$.

COMPASS-31 scores and autoantibody status. Euroimmun immunoblot assays to detect autoantibody profiles were performed on patients with available serum samples (83/104 patients, $80 \%$ ). Among patients with complete autoantibody data, 21 patients (25\%) had anti-centromere proteins (CENP) antibodies, 31 patients (37\%) had anti-topoisomerase I (topo I) antibodies, and 19 patients (23\%) had anti-RNA pol 3 antibodies. Patients with anti-CENP antibodies had significantly higher total COMPASS-31 scores compared to anti-CENP-negative patients $(30.7 \pm 17.9$ vs $21.3 \pm 13.8$, $\mathrm{p}=0.04)$. This difference remained after adjustment for age, sex, and race $(\beta 4.3, \mathrm{CI} 0.3-8.3, \mathrm{p}=0.04)$, but lost statistical significance after disease duration was added to the model $(\beta 2.6, \mathrm{CI}-1.5$ to $6.6, \mathrm{p}=0.21)$. Higher COMPASS-31 scores among anti-CENP-positive patients were driven by higher scores in vasomotor dysfunction, GI dysfunction, and secretomotor dysfunction ( $\mathrm{p}<0.05$ for all comparisons). There was no difference in COMPASS-31 scores between patients with and without anti-topo 1 antibodies. Patients with anti-RNA pol 3 antibodies had lower total COMPASS-31 scores $(17.9 \pm 12.4$ vs $25.4 \pm 15.8, \mathrm{p}=0.04)$, although this lost significance after adjustment for age, sex, and race $(\beta-3.5, \mathrm{CI}-7.7$ to $0.7, \mathrm{p}=0.10$; Table 3$)$.

COMPASS-31 scores and clinical associations in SSc. We found that women with SSc had significantly higher mean COMPASS- 31 scores than men $(26.1 \pm 15.1$ vs $15.3 \pm 16.0$, $\mathrm{p}=0.04)$. Disease duration was positively associated with

Personal non-commercial use only. The Journal of Rheumatology Copyright $\subset$ $\odot 2018$. All rights reserved. 
Table 2. COMPASS-31 questionnaire scores and subdomain scores in SSc compared to previously published healthy controls ( $\mathrm{n}=30$ ) and patients with other diseases that present with autonomic symptoms. Data are presented as mean (SD).

\begin{tabular}{|c|c|c|c|c|c|}
\hline Subdomain & $\mathrm{SSc}$ & Healthy Controls & $\begin{array}{c}\text { Fibromyalgia, } \\
n=30\end{array}$ & $\begin{array}{l}\text { Diabetic Polyneuropathy, } \\
\qquad \mathrm{n}=29\end{array}$ & $\begin{array}{c}\text { Small-fiber } \\
\text { Polyneuropathy, } \mathrm{n}=66\end{array}$ \\
\hline Orthostatic & $8.2(9.3)$ & $2.9(6.4)$ & $9.2(8.5)$ & $11.2(12.4)$ & $14.0(12.4)$ \\
\hline Vasomotor & $2.6(1.4)$ & $0.1(0.3)$ & $0.8(1.3)$ & $1.0(1.4)$ & $1.6(2.1)$ \\
\hline Secretomotor & $4.8(3.3)$ & $1.1(2.4)$ & $4.9(3.2)$ & $5.5(3.6)$ & $5.4(6.9)$ \\
\hline Gastrointestinal & $7.7(4.9)$ & $3.1(2.7)$ & $8.4(4.4)$ & $8.1(4.8)$ & $6.6(5.4)$ \\
\hline Urinary & $0.9(1.3)$ & $0.5(0.9)$ & $1.8(1.6)$ & $1.6(2.0)$ & $1.0(1.6)$ \\
\hline Pupillomotor & $1.6(1.1)$ & $1.2(0.8)$ & $2.4(1.1)$ & $2.2(1.3)$ & $1.7(1.4)$ \\
\hline Total COMPASS-31 & $24.9(15.5)$ & $8.9(8.7)$ & $27.5(14.2)$ & $28.9(19.1)$ & $30.2(23.2)$ \\
\hline
\end{tabular}

SSc: systemic sclerosis.

Table 3. Mean COMPASS-31 questionnaire scores by autoantibody for the 83 systemic sclerosis (SSc) patients with complete Euroimmun autoantibody profiles.

\begin{tabular}{|c|c|c|c|c|c|}
\hline Autoantibody Subtype & $\mathrm{n} / \mathrm{N}(\%)$ & $\begin{array}{c}\text { Mean Compass } \\
\text { Score (SD) }\end{array}$ & $\mathrm{p}$ & $\begin{array}{l}\text { Adjusted Model 1: } \\
\text { Age, Sex, Race }\end{array}$ & $\begin{array}{l}\text { Adjusted Model 2: } \\
\text { Model } 1+\text { Disease Duration }\end{array}$ \\
\hline \multicolumn{6}{|l|}{ Anti-centromere } \\
\hline Positive & $21 / 83(25)$ & 30.7 (17.9) & 0.04 & $\beta 4.3(0.3-8.3), p=0.04$ & $\beta 2.6(-1.5$ to 6.6$), p=0.21$ \\
\hline Negative & $62 / 83(75)$ & $21.3(13.8)$ & & & \\
\hline \multicolumn{6}{|l|}{ Anti-topoisomerase I } \\
\hline Positive & $31 / 83(37)$ & $21.2(13.6)$ & 0.24 & $\beta-2.5(-6.2$ to 1.2$), p=0.19$ & $\beta-1.7(-5.3$ to 1.8$), p=0.34$ \\
\hline Negative & $52 / 83(63)$ & $25.1(16.3)$ & & & \\
\hline \multicolumn{6}{|c|}{ Anti-RNA polymerase III } \\
\hline Positive & $19 / 83(23)$ & $17.9(12.4)$ & 0.04 & $\beta-3.5(-7.7$ to 0.7$), p=0.10$ & $\beta-2.3(-6.4$ to 1.7$), p=0.26$ \\
\hline Negative & $64 / 83(77)$ & $25.4(15.8)$ & & & \\
\hline
\end{tabular}

Student $\mathrm{t}$ test was used to compare COMPASS-31 scores between patients with and without each autoantibody. Multivariable linear regression analysis was used to adjust for age, sex, and race (Model 1) and age, sex, race, and disease duration (Model 2). Data are presented as the $\beta$ coefficient and the $95 \%$ CI.

total COMPASS-31 score [predicted COMPASS-31 $=0.5 \times$ disease duration $\left.(\mathrm{yrs})+18.5, \mathrm{R}^{2}=0.07, \mathrm{p}<0.01\right]$, whereas there was no association between COMPASS-31 score and age $(\mathrm{p}>0.05)$. There was no difference in mean COMPASS-31 scores by patient race (white vs African American) or SSc subtype (limited vs diffuse), and there was no association with resting heart rate. Patients with dry mouth $(27.3 \pm 15.9$ vs $18.3 \pm 12.6, \mathrm{p}<0.01)$, arthralgias $(26.0 \pm 15.7$ vs $16.3 \pm 11.3, \mathrm{p}<0.05)$, and significant GI disease $(29.1 \pm$ 16.6 vs $18.2 \pm 10.8, \mathrm{p}<0.0001)$ had higher mean COMPASS-31 scores than patients without these clinical findings.

GI disease in SSc is associated with higher COMPASS-31 scores. To determine whether GI disease in SSc may be part of a dysautonomia syndrome, we investigated whether patients with GI disease had other symptoms of autonomic dysfunction. For this analysis, we compared the COMPASS-31 subdomain scores of patients with significant GI disease to those with absent or mild GI disease. Of our total study cohort, 64 patients $(62 \%)$ had significant GI disease, which was defined as a Medsger severity score $\geq 2$. Compared to patients with mild or absent GI disease, patients with significant GI disease had higher scores in orthostatic intolerance [median 10.0 (IQR 0-20.0) vs 0 (IQR 0-12.0), p = 0.006] and secretomotor dysfunction [median 6.4 (IQR 2.7-8.6) vs 4.3 (IQR 0.5-6.4), p = 0.03; Figure 1A-B). There was a higher burden of autonomic gastrointestinal symptoms among patients with more significant GI disease compared to patients with mild or absent GI disease $(8.2 \pm 4.5$ vs $4.8 \pm$ $3.3, p<0.001)$. We found no relationship between GI disease severity and scores in vasomotor, urinary, or pupillomotor dysfunction. We then constructed a multivariable linear regression model to adjust for potential clinical confounders (age, sex, race, and disease duration). After adjustment for these covariates, the positive association remained between significant GI disease and total COMPASS-31 score ( $\beta 4.8$, CI $1.6-8.1, \mathrm{p}=0.004)$ and orthostatic intolerance score $(\beta 2.4, \mathrm{CI} 0.4-4.4, \mathrm{p}=0.02)$. The association between significant GI disease and secretomotor dysfunction scores remained after adjustment for age, sex, and race $(\beta 0.8, \mathrm{CI}$ $0.1-1.4, \mathrm{p}=0.03)$, but lost statistical significance when disease duration was added to the model $(\mathrm{p}>0.5)$.

We then examined whether a dose-response relationship exists between the severity of GI disease and autonomic symptom burden. We divided our cohort into 3 groups based on the Medsger GI disease severity score: absent or mild GI disease (Medsger severity score $0-1, n=40$ ), significant GI disease (Medsger severity score of 2, n = 57), and severe GI 
A)

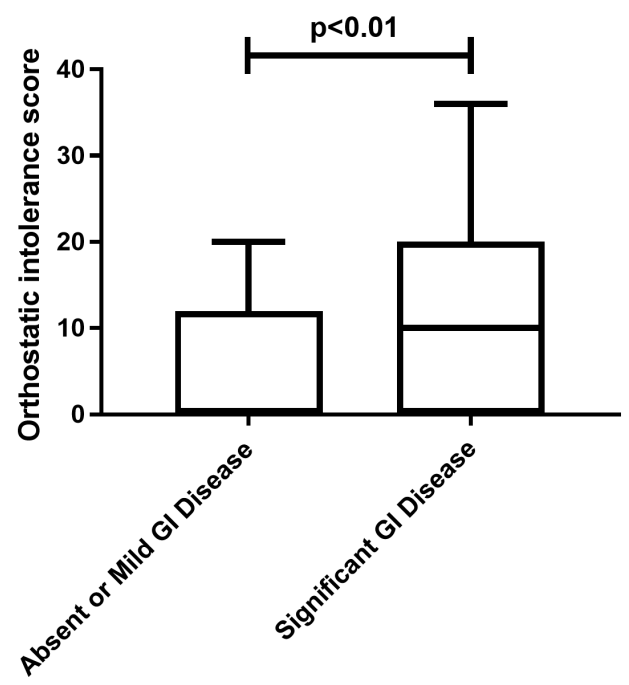

B)

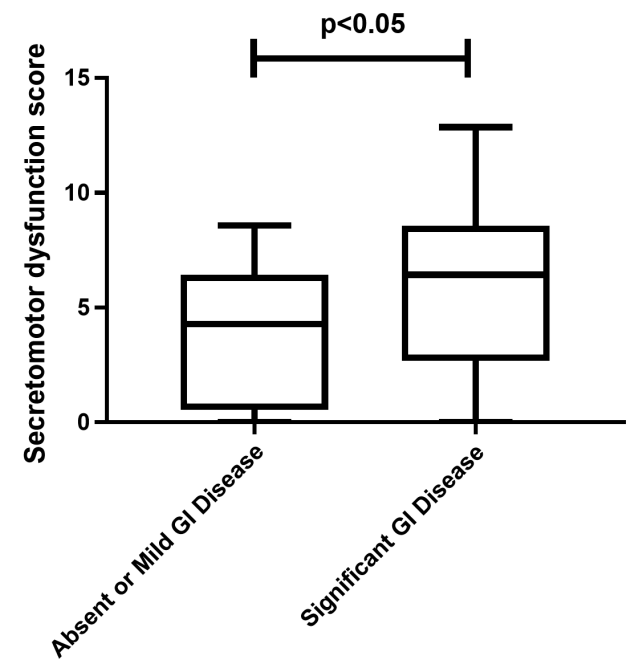

C)

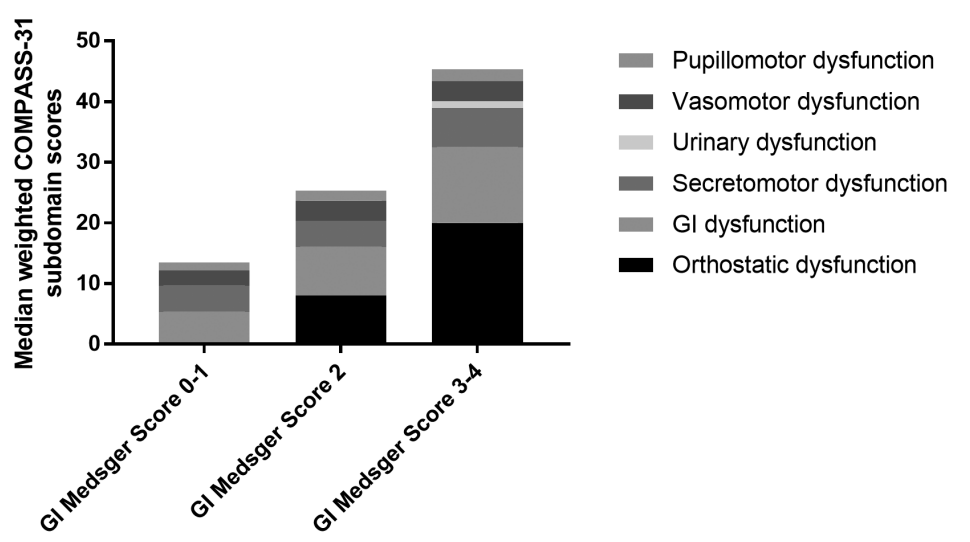

D)

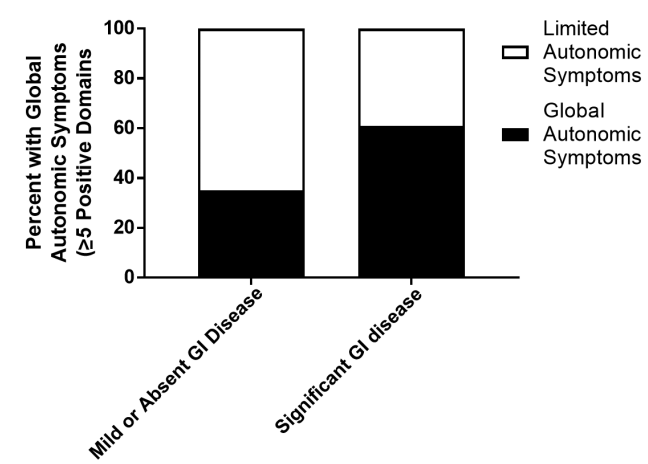

E)

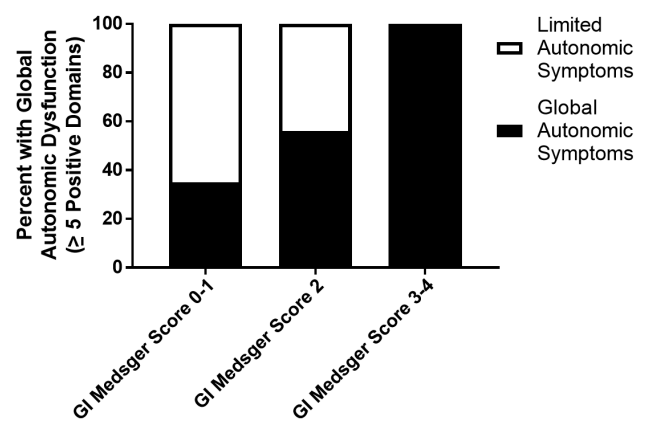

Figure 1. Relationship between GI disease severity and autonomic symptoms in patients with systemic sclerosis (SSc). Patients with SSc and significant gastrointestinal (GI) disease (Medsger severity score $\geq 2$ ) have increased median (A) orthostatic intolerance scores $(\mathrm{p}=0.006)$ and $(\mathrm{B})$ secretomotor dysfunction scores $(\mathrm{p}=0.03)$ compared to patients with absent or mild GI disease. (C) Median COMPASS-31 scores across various subdomains increase as GI disease severity increases. (D) A higher percent of patients with significant GI disease have global autonomic symptoms (defined as $\geq 5$ positive subdomains of the COMPASS-31) compared to patients with mild or absent GI disease $(\mathrm{p}=0.01)$. (E) There is a dose-response relationship between GI disease severity and global autonomic symptoms. COMPASS-31: Composite Autonomic Symptom Score-31 questionnaire. 
disease (Medsger severity score of 3-4, $n=7$ ). We found that autonomic symptom burden across all subdomains of the COMPASS- 31 increased as the severity of GI disease increased (Figure 1C).

GI disease in SSc is associated with symptoms of global autonomic dysfunction. To further explore the relationship between GI disease in SSc and symptoms of global dysautonomia, we divided the cohort into 2 predefined groups based on the number of positive subdomains (score $>0$ ) within the COMPASS-31. Fifty-three patients $(51 \%)$ had $\geq 5$ positive subdomains and were defined as having global autonomic symptoms, and 51 patients $(49 \%)$ had $<5$ positive subdomains and were defined as having limited autonomic symptoms. We found that more patients with significant GI disease (Medsger score $\geq 2$ ) had global autonomic symptoms compared to patients with mild or absent GI disease (Medsger score $<2 ; 61 \%$ vs 35\%, $p=0.01$; Figure 1D). The association between significant GI disease and the presence of global autonomic symptoms remained after adjusting for age, sex, race, and disease duration (OR 3.2, 95\% CI 1.3-8.4, $\mathrm{p}=0.01)$. We also found that the percent of patients who report symptoms in $\geq 5$ subdomains of the COMPASS-31 increases as the severity of GI disease increases (Figure 1E).

SSc patients with myopathy have more symptoms of global autonomic dysfunction. Because our previous study identified an association between severe GI disease and myopathy in $\mathrm{SSc}^{24}$, we next examined the relationship between myopathy and symptoms of dysautonomia. We identified higher orthostatic intolerance scores [median 14.0 (IQR 2.0-20.0) vs 0 (IQR 0-16.0), $\mathrm{p}=0.02]$ and a trend toward higher total COMPASS-31 scores $(32.3 \pm 16.4$ vs $23.5 \pm 15.1, \mathrm{p}=0.06)$ among patients with myopathy compared to patients without myopathy. There was no relationship between the presence of myopathy and subdomain scores in vasomotor, secretomotor, GI, urinary, or pupillomotor dysfunction ( $\mathrm{p}>0.05)$. When patients were grouped by the number of positive subdomain scores, more patients with myopathy had global autonomic symptoms ( $\geq 5$ positive subdomains) compared to patients without myopathy ( $75 \%$ vs $47 \%, \mathrm{p}=0.03$; Figure 2 ). We did not pursue multivariable modeling given the low number of patients with myopathy $(n=16)$.

\section{DISCUSSION}

We administered the COMPASS-31 questionnaire to a well-characterized cohort of patients with SSc to quantify symptoms of autonomic dysfunction and to determine clinical risk factors for dysautonomia. To our knowledge, this is the first time that a validated autonomic dysfunction questionnaire has been used to measure global autonomic symptom burden across a large population of patients with SSc. Consistent with prior studies $7,9,10,25$, we found that patients with SSc have a high burden of autonomic symptoms that involve many aspects of the autonomic nervous system. Resting heart rate is a poor predictor of autonomic symptom burden in SSc, and our findings support the use of patient-reported symptom scores in screening for autonomic dysfunction.

Among the clinical features of SSc that we analyzed, we found a strong association between the presence of GI disease and higher COMPASS-31 scores. We considered whether this finding occurred because questions about GI dysfunction are a component of the COMPASS-31 questionnaire. However, positive responses for GI dysfunction in the questionnaire were not solely responsible for this association. Patients with

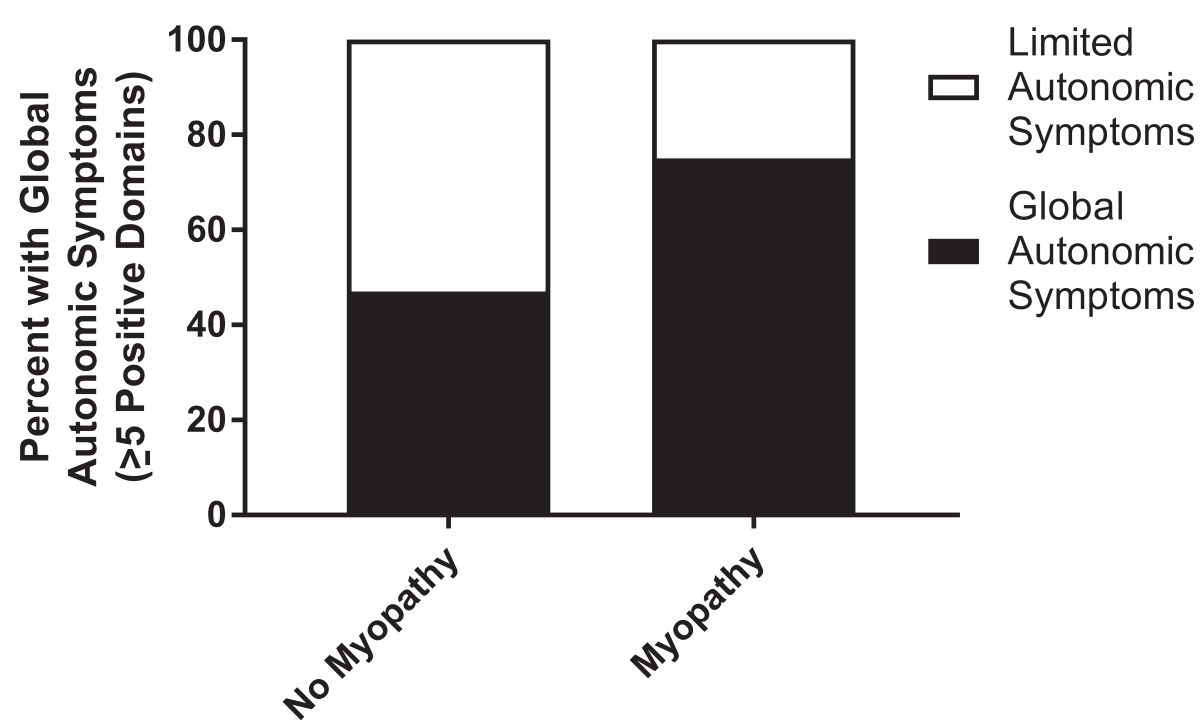

Figure 2. Comparison of autonomic symptom burden in systemic sclerosis patients with and without myopathy. Patients with myopathy are more likely to have global autonomic symptoms (defined as $\geq 5$ positive domains) compared to patients without myopathy $(\mathrm{p}=0.03)$. 
significant GI disease frequently reported symptoms across many facets of the autonomic nervous system (e.g., orthostatic intolerance and secretomotor dysfunction), suggestive of global dysautonomia. Our findings are also consistent with previous studies that suggested that early GI disease in SSc may be mediated by dysfunction of the autonomic nervous system $^{26}$, and raise the possibility that GI disease in SSc may be a cause or consequence of autonomic dysfunction.

Autonomic symptoms are common in other rheumatic diseases associated with GI dysmotility, including Sjögren syndrome and systemic lupus erythematosus ${ }^{27,28,29}$, suggesting that pathology of the autonomic nervous system may be a common clinical feature among certain patient subsets. While the mechanism of dysautonomia in these patients remains unclear, the cause of autoimmune GI dysmotility in other patient groups, such as those with autoimmune autonomic ganglionopathy, is well established. In this autoimmune disease, GI dysmotility and other symptoms of autonomic dysfunction result from pathogenic autoantibodies targeting ganglionic acetylcholine receptors ${ }^{30}$. More research is needed in SSc and other rheumatic diseases to determine whether similar pathogenic antibodies targeting components of the autonomic nervous system can be identified.

We observed a trend toward increased global dysautonomia symptoms among SSc patients with myopathy. Although these findings require further confirmation because of the low number of patients with myopathy in the cohort, these data are consistent with data from our group and a study by Nishimagi and colleagues, which demonstrated an association between myopathy and GI disease in $\mathrm{SSc}^{24,31}$. Future studies are also needed to explore the role of the autonomic nervous system in patients with myopathy and SSc.

Notable strengths of our study include the use of a diverse and well-characterized cohort of patients and the use of a validated questionnaire that assesses various aspects of the autonomic nervous system. Regarding study limitations, we did not have a healthy control group and therefore compared our findings with other relevant cohorts with COMPASS-31 scores in the literature. It is conceivable that the differences in COMPASS-31 scores observed were unrelated to SSc, but instead resulted from demographic differences that we were unable to control for. However, both our SSc cohort and the healthy controls were demographically similar, consisting predominately of white middle-aged women. We also had very few patients with myopathy, so our results regarding these patients are tentative and need to be repeated with a larger sample size. We were unable to investigate the mental health of participants in our cohort study, although mental health may be impaired in $\mathrm{SSc}^{32,33}$ and has been shown to affect COMPASS-31 scores in other diseases ${ }^{34}$. Future studies of autonomic dysfunction in SSc should explore what role mental health and fatigue have in mediating the observed associations. Lastly, the COMPASS-31 questionnaire measures symptoms of dysautonomia rather than objective measures of autonomic dysfunction. It can be challenging to distinguish some symptoms of autonomic dysfunction from features of SSc itself, particularly regarding RP and GI symptoms such as constipation, early satiety, and bloating. Although this is an inherent limitation, the substantial overlap in symptoms of SSc and global dysautonomia may provide important insights into the role of autonomic dysfunction in the pathogenesis of SSc.

Our findings provide important insights into the association between symptoms of dysautonomia and the dysfunction of specific organ systems in patients with SSc. Further mechanistic studies investigating the role of autonomic dysfunction in SSc, and how it relates to disease biology, are warranted.

\section{REFERENCES}

1. Akesson A, Wollheim FA. Organ manifestations in 100 patients with progressive systemic sclerosis: a comparison between the CREST syndrome and diffuse scleroderma. Br J Rheumatol 1989;28:281-6.

2. Prescott RJ, Freemont AJ, Jones CJ, Hoyland J, Fielding P. Sequential dermal microvascular and perivascular changes in the development of scleroderma. J Pathol 1992;166:255-63.

3. Varga J, Abraham D. Systemic sclerosis: a prototypic multisystem fibrotic disorder. J Clin Invest 2007;117:557-67.

4. Sonnex C, Paice E, White AG. Autonomic neuropathy in systemic sclerosis: a case report and evaluation of six patients. Ann Rheum Dis 1986;45:957-60.

5. Dessein PH, Gledhill RF. More on autonomic neuropathy in systemic sclerosis. Ann Rheum Dis 1988;47:261-3.

6. Bertinotti L, Bracci S, Nacci F, Colangelo N, Del Rosso A, Casale $\mathrm{R}$, et al. The autonomic nervous system in systemic sclerosis. A review. Clin Rheumatol 2004;23:1-5.

7. Kahaleh B, Matucci Cerinic M. Raynaud's phenomenon and scleroderma dysregulated neuroendothelial control of vascular tone Arthritis Rheum 1995;38:1-4.

8. Di Franco M, Paradiso M, Riccieri V, Basili S, Mammarella A, Valesini G. Autonomic dysfunction and microvascular damage in systemic sclerosis. Clin Rheumatol 2007;26:1278-83.

9. Iovino P, Valentini G, Ciacci C, De Luca A, Tremolaterra F, Sabbatini F, et al. Proximal stomach function in systemic sclerosis: relationship with autonomic nerve function. Dig Dis Sci 2001;46:723-30.

10. Lock G, Straub RH, Zeuner M, Antoniou E, Holstege A, Schölmerich J, et al. Association of autonomic nervous dysfunction and esophageal dysmotility in systemic sclerosis. J Rheumatol 1998;25:1330-5.

11. Dessein PH, Joffe BI, Metz RM, Millar DL, Lawson M, Stanwix AE. Autonomic dysfunction in systemic sclerosis: Sympathetic overactivity and instability. Am J Med 1992;93:143-50.

12. Cerinic MM, Generini S, Pignone A, Casale R. The nervous system in systemic sclerosis (scleroderma). Clinical features and pathogenetic mechanisms. Rheum Dis Clin North Am 1996; 22:879-92.

13. Pawlowski A. The nerve network of the skin in diffuse scleroderma and clinically similar conditions. Arch Dermatol 1963;88:868-73.

14. Ormea F. [Comparative studies of the peripheral autonomous nervous system in diffuse sclerodermia and in connective tissue changed by various skin diseases]. [Article in Italian] Dermatologica 1952;105:8-17.

15. Sletten DM, Suarez GA, Low PA, Mandrekar J, Singer W COMPASS 31: a refined and abbreviated Composite Autonomic Symptom Score. Mayo Clin Proc 2012;87:1196-201. 
16. Suarez GA, Opfer-Gehrking TL, Offord KP, Atkinson EJ, O'Brien PC, Low PA. The Autonomic Symptom Profile: a new instrument to assess autonomic symptoms. Neurology 1999;52:523-8.

17. Treister R, O'Neil K, Downs HM, Oaklander AL. Validation of the composite autonomic symptom scale 31 (COMPASS-31) in patients with and without small fiber polyneuropathy. Eur J Neurol 2015;22:1124-30.

18. Cortez MM, Nagi Reddy SK, Goodman B, Carter JL, Wingerchuk DM. Autonomic symptom burden is associated with MS-related fatigue and quality of life. Mult Scler Relat Disord 2015;4:258-63.

19. Greco C, Di Gennaro F, D'Amato C, Morganti R, Corradini D, Sun A, et al. Validation of the Composite Autonomic Symptom Score 31 (COMPASS 31) for the assessment of symptoms of autonomic neuropathy in people with diabetes. Diabet Med 2017;34:834-8.

20. Vincent A, Whipple MO, Low PA, Joyner M, Hoskin TL. Patients with fibromyalgia have significant autonomic symptoms but modest autonomic dysfunction. PM R 2016;8:425-35.

21. LeRoy EC, Medsger TA. Criteria for the classification of early systemic sclerosis. J Rheumatol 2001;28:1573-6.

22. Medsger TA, Bombardieri S, Czirjak L, Scorza R, Della Rossa A, Bencivelli W. Assessment of disease severity and prognosis. Clin Exp Rheumatol 2003;21:S42-6.

23. Patterson KA, Roberts-Thomson PJ, Lester S, Tan JA, Hakendorf P, Rischmueller M, et al. Interpretation of an extended autoantibody profile in a well-characterized Australian systemic sclerosis (scleroderma) cohort using principal components analysis. Arthritis Rheumatol 2015;67:3234-44.

24. McMahan ZH, Paik JJ, Wigley FM, Hummers LK. Determining the risk factors and clinical features associated with severe gastrointestinal dysmotility in systemic sclerosis. Arthritis Care Res 2017 Nov 28 (E-pub ahead of print).

25. Ferri C, Emdin M, Giuggioli D, Carpeggiani C, Maielli M, Varga A, et al. Autonomic dysfunction in systemic sclerosis: time and frequency domain 24 hour heart rate variability analysis. $\mathrm{Br} \mathrm{J}$ Rheumatol 1997;36:669-76.
26. Amaral TN, Peres FA, Lapa AT, Marques-Neto JF, Appenzeller S. Neurologic involvement in scleroderma: A systematic review. Semin Arthritis Rheum 2013;43:335-47.

27. Newton JL, Frith J, Powell D, Hackett K, Wilton K, Bowman S, et al. Autonomic symptoms are common and are associated with overall symptom burden and disease activity in primary Sjögren's syndrome. Ann Rheum Dis 2012;71:1973-9.

28. Evrengl H, Dursunoglu D, Cobankara V, Polat B, Seleci D, Kabuku $\mathrm{S}$, et al. Heart rate variability in patients with rheumatoid arthritis. Rheumatol Int 2004;24:198-202.

29. Stojanovich L, Milovanovich B, de Luka SR, PopovichKuzmanovich D, Bisenich V, Djukanovich B, et al. Cardiovascular autonomic dysfunction in systemic lupus, rheumatoid arthritis, primary Sjögren syndrome and other autoimmune diseases. Lupus 2007;16:181-5.

30. Vernino S, Low PA, Fealey RD, Stewart JD, Farrugia G, Lennon VA. Autoantibodies to ganglionic acetylcholine receptors in autoimmune autonomic neuropathies. N Eng1 J Med 2000; 343:847-55,

31. Nishimagi E, Tochimoto A, Kawaguchi Y, Satoh T, Kuwana M, Takagi K, et al. Characteristics of patients with early systemic sclerosis and severe gastrointestinal tract involvement. J Rheumatol 2007;34:2050-5.

32. Mozzetta A, Antinone V, Alfani S, Neri P, Bonda PF, Pasquini P, et al. Mental health in patients with systemic sclerosis: a controlled investigation. J Eur Acad Dermatology Venereol 2008;22:336-40.

33. Thombs BD, Bassel M, McGuire L, Smith MT, Hudson M, Haythornthwaite JA. A systematic comparison of fatigue levels in systemic sclerosis with general population, cancer and rheumatic disease samples. Rheumatology 2008;47:1559-63.

34. Mohammed J, Derom E, De Wandele I, Rombaut L, Calders P. Autonomic symptoms in patients with moderate and severe chronic obstructive pulmonary disease. Acta Clin Belg 2018;73:182-90. 